TRANSACTIONS OF THE

AMERICAN MATHEMATICAL SOCIETY

Volume 353, Number 9, Pages 3511-3529

S 0002-9947(01)02768-4

Article electronically published on April 24, 2001

\title{
THE FINITE VERTEX-PRIMITIVE AND VERTEX-BIPRIMITIVE $s$-TRANSITIVE GRAPHS FOR $s \geq 4$
}

\author{
CAI HENG LI
}

\begin{abstract}
A complete classification is given for finite vertex-primitive and vertex-biprimitive $s$-transitive graphs for $s \geq 4$. The classification involves the construction of new 4-transitive graphs, namely a graph of valency 14 admitting the Monster simple group $\mathrm{M}$, and an infinite family of graphs of valency 5 admitting projective symplectic groups $\operatorname{PSp}(4, p)$ with $p$ prime and $p \equiv \pm 1(\bmod 8)$. As a corollary of this classification, a conjecture of Biggs and Hoare (1983) is proved.
\end{abstract}

\section{INTRODUCTION}

Denote by $\Gamma$ a finite connected graph with vertex set $V \Gamma$ and edge set $E \Gamma$. For a positive integer $s$, an $s$-arc of $\Gamma$ is an $(s+1)$-tuple $\left(v_{0}, v_{1}, \ldots, v_{s}\right)$ of vertices such that $\left\{v_{i-1}, v_{i}\right\} \in E \Gamma$ for $1 \leq i \leq s$ and $v_{i-1} \neq v_{i+1}$ for $1 \leq i \leq s-1$. Let Aut $\Gamma$ denote the full automorphism group of $\Gamma$. If $G \leq$ Aut $\Gamma$ and $G$ is transitive on $V \Gamma$ and on the set of $s$-arcs of $\Gamma$, then $\Gamma$ is called a $(G, s)$-arc transitive graph; while if in addition $G$ is not transitive on the set of $(s+1)$-arcs of $\Gamma$, then $\Gamma$ is called a $(G, s)$-transitive graph. In particular, if $G=$ Aut $\Gamma$, then a $(G, s)$-arc transitive or $(G, s)$-transitive graph is simply called an $s$-arc transitive graph or an $s$-transitive graph, respectively. (We remark that for any positive integer $s$ a cycle is $(s+1)$-arc transitive so that it is not $s$-transitive, so the valency of an $s$-transitive graph is greater than 2.) The main purpose of this paper is to classify finite vertex-primitive and vertex-biprimitive $s$-transitive graphs for $s \geq 4$.

Interest in $s$-transitive graphs stems from a beautiful result of Tutte (1947) who proved that there exist no finite $s$-transitive cubic graphs for $s \geq 6$. Tutte's Theorem was generalized by Weiss (1981) who proved that there exist no finite $s$-transitive graphs for $s=6$ and $s \geq 8$. Since then, $s$-transitive graphs have received considerable attention in the literature (see, for example, [11, 13, 24, 34]), and investigating the following problem has become one of the central topics in algebraic graph theory:

Problem. Construct and characterize $s$-transitive graphs for $s \geq 2$, and in particular, for $s=4,5$ and 7 .

It is known that for each value of $s \in\{1,2,3,4,5,7\}$, there exist $s$-transitive graphs (see Section 2). However, it is widely believed that $s$-transitive graphs for

Received by the editors November 12, 1999 and, in revised form, July 11, 2000.

2000 Mathematics Subject Classification. Primary 05C25, $20 \mathrm{~B} 05$.

This work forms a part of an ARC project and is supported by an ARC Fellowship.

The author is grateful to C.E. Praeger, A.A. Ivanov and R. Weiss for their helpful comments on the work, and to the referee for constructive suggestions. 
large values of $s$, that is $s \in\{4,5,7\}$, are rare and hard to construct. An $s$-transitive graph is said to be basic if it is not a normal cover of a smaller $s$-transitive graph (see [26]). It was shown by Conway that any $s$-transitive graph has a nontrivial $s$-transitive normal cover (see [3, Section 19]). This suggests that the study of $s$-transitive graphs should be divided into two steps: studying basic $s$-transitive graphs and studying their normal covers (see [26]). Using such an approach, it is shown in [18, 19] that the order of a finite $s$-transitive graph for $s \geq 4$ is neither a prime-power nor an odd integer.

Let $s$ be an integer in $\{4,5,7\}$. Up to now, the only known basic $s$-transitive graphs consist of the following graphs (see Sections 2 and 3): several infinite families of $s$-transitive graphs of valency 3 and valency 4 , constructed in [4, 6, 7, 39]; the classical generalized $(s-1)$-gon graphs which are bipartite; and three graphs from sporadic simple groups: the Janko group $\mathrm{J}_{3}$, the Rudvalis group $\mathrm{Ru}$ and the Thompson group $\mathrm{Th}$, which were constructed in [37] and [28]. The $\mathrm{J}_{3}$-graph has valency 5 ; the $\mathrm{Ru}$-graph and the Th-graph have valency 6 . These three graphs were the only known non-bipartite $s$-transitive graphs for $s \geq 4$ of valency greater than 4 until now. Here we construct a 4-transitive graph of valency 14 from the Monster sporadic simple group, and an infinite family of 4 -transitive graphs of valency 5 from the projective symplectic groups $\operatorname{PSp}(4, p)$ with $p$ prime.

Theorem 1.1. There exists exactly one 4-transitive graph $\Gamma$ such that $\Gamma$ is vertexprimitive and Aut $\Gamma=\mathrm{M}$, the Monster simple group. Further, the graph $\Gamma$ has valency 14 .

The Monster-graph is the only known non-bipartite basic $s$-transitive graph of valency greater than 6 , for $s \geq 4$.

Theorem 1.2. For each prime $p \equiv \pm 1(\bmod 8)$, there exists exactly one 4-transitive graph $\Gamma$ such that $\Gamma$ is vertex-primitive and Aut $\Gamma=\operatorname{PSp}(4, p)$. Further, $\Gamma$ has valency 5 .

The PSp $(4, p)$-graphs form the only known infinite family of basic $s$-transitive graphs of valency greater than 4 , for $s \geq 4$.

Moreover, it will be shown that the Monster-graph and the $\operatorname{PSp}(4, p)$-graphs, together with some other known graphs, form the class of vertex-primitive or vertexbiprimitive $s$-transitive graphs for $s \geq 4$. (A finite graph $\Gamma$ is said to be vertexprimitive if Aut $\Gamma$ acts primitively on $V \Gamma$; while a bipartite graph $\Gamma$ with bi-parts $\Delta_{1}$ and $\Delta_{2}$ is said to be vertex-biprimitive if the setwise stabilizer (Aut $\left.\Gamma\right)_{\Delta_{i}}$ is primitive on $\Delta_{i}$.)

For two groups $G$ and $F$, let $G$.F denote an extension of $G$ by $F$, and $G \rtimes F$ denote a semidirect product of $G$ by $F$. For a positive integer $n$, as in the Atlas [8], denote by $[n]$ a group of order $n$ throughout the paper. Given a finite group $G$, a core-free subgroup $H$ of $G$ and a 2-element $g \in G \backslash H$ with $g^{2} \in H$, the coset graph $\Gamma=\Gamma(G, H, H g H)$ is the graph with vertex set $[G: H]$, the set of right cosets of $H$ in $G$, such that $H x, H y$ are adjacent if and only if $y x^{-1} \in H g H$ (see Section 2 for more detailed discussions). The next theorem gives a complete classification of finite vertex-primitive $s$-transitive graphs for $s \in\{4,5,7\}$.

Theorem 1.3. Let $k, s$ be positive integers, and assume that $s \geq 4$. A finite graph $\Gamma$ of valency $k$ is vertex-primitive and s-transitive if and only if $\Gamma \cong \Gamma(G, H, H g H)$, where $G, H, k, s$ are as listed in Table 1 . Further, such a graph $\Gamma$ is uniquely determined by its order $|V \Gamma|$. 
TABLE 1.

\begin{tabular}{|c|c|c|c|c|}
\hline Aut $\Gamma=G$ & $H$ & $k$ & $s$ & reference \\
\hline \hline $\begin{array}{c}\mathrm{PSL}(2, p) \\
p \equiv \pm 1(\bmod 16) \text { prime }\end{array}$ & $\mathrm{S}_{4}$ & 3 & 4 & Wong (1967) \\
\hline $\mathrm{PSL}(3,3) . \mathbb{Z}_{2}$ & $\mathrm{~S}_{4} \times \mathbb{Z}_{2}$ & 3 & 5 & Wong (1967) \\
\hline $\begin{array}{c}\mathrm{PSp}(4, p) \\
p \equiv \pm 1(\bmod 8) \text { prime }\end{array}$ & $\mathbb{Z}_{2}^{4} \rtimes \mathrm{GL}(2,4)$ & 5 & 4 & Theorem[1.2 \\
\hline $\mathrm{J}_{3}$ & $\mathbb{Z}_{2}^{4} \rtimes \mathrm{GL}(2,4)$ & 5 & 4 & Weiss (1986) \\
\hline $\mathrm{Ru}$ & $\mathbb{Z}_{5}^{2} \rtimes \mathrm{GL}(2,5)$ & 6 & 4 & Stroth \& Weiss (1990) \\
\hline $\mathrm{Th}$ & $\mathbb{Z}_{5}^{2} \rtimes \mathrm{GL}(2,5)$ & 6 & 4 & Stroth \& Weiss (1990) \\
\hline $\mathrm{M}$ & $\mathbb{Z}_{13}^{2} \rtimes\left(\mathbb{Z}_{4} . \mathrm{PGL}(2,13)\right)$ & 14 & 4 & Theorem[1.1 \\
\hline
\end{tabular}

TABLE 2 .

\begin{tabular}{|c|c|c|c|c|c|}
\hline$T=\operatorname{soc}(G)$ & $G$ & $H \cap T$ & $k$ & $s$ & reference \\
\hline \hline $\operatorname{PSL}(3, q)$ & $G \leq \operatorname{Aut}(T)$ & $\begin{array}{c}{\left[q^{2}\right] \rtimes\left(\mathbb{Z}_{l} \text {. PGL }(2, q)\right)} \\
\text { where } l=\frac{q-1}{(q-1,3)}\end{array}$ & $q+1$ & 4 & Section 2 \\
\hline $\operatorname{Sp}\left(4,2^{m}\right)$ & $G \leq \operatorname{Aut}(T)$ & {$\left[2^{3 m}\right] \rtimes \mathrm{GL}\left(2,2^{m}\right)$} & $2^{m}+1$ & 5 & Section 2 \\
\hline $\mathrm{G}_{2}\left(3^{m}\right)$ & $G \leq \operatorname{Aut}(T)$ & {$\left[3^{5 m}\right] \rtimes \mathrm{GL}\left(2,3^{m}\right)$} & $3^{m}+1$ & 7 & Section 2 \\
\hline $\begin{array}{c}\mathrm{PSL}(2, p) \\
p \equiv \pm 1(\bmod 8)\end{array}$ & $\mathrm{PGL}(2, p)$ & $H=\mathrm{S}_{4}$ & 3 & 4 & Biggs \& Hoare 4 \\
\hline $\mathrm{A}_{6}$ & $\mathrm{P \Gamma L}(2,9)$ & $H=\mathrm{S}_{4} \times \mathbb{Z}_{2}$ & 3 & 5 & Tutte's 8-Cage \\
\hline $\mathrm{M}_{12}$ & $\mathrm{M}_{12}, \mathbb{Z}_{2}$ & $H=\mathbb{Z}_{3}^{2} \rtimes \mathrm{GL}(2,3)$ & 4 & 4 & Weiss (1985) \\
\hline
\end{tabular}

Remark. The $\mathrm{J}_{3}$-graph was used to characterize the Janko simple group $\mathrm{J}_{3}$ by Weiss [37]; the Ru-graph was used to characterize the Rudvalis simple group Ru by Stroth and Weiss [28].

Some vertex-biprimitive $s$-transitive graphs are covers of vertex-primitive $s$ transitive graphs. For a $(G, s)$-transitive graph $\Sigma=\Gamma(G, H, H g H)$, let $F=G \times\langle z\rangle$ where $z$ is an involution, and let $\Gamma=\Gamma(F, H, H g z H)$. Then $\Gamma$ is an $(F, s)$-transitive bipartite graph, and is called the standard double cover of $\Sigma$. The following theorem gives a complete classification of finite vertex-biprimitive $s$-transitive graphs for $s \in\{4,5,7\}$.

Theorem 1.4. Let $k, s$ be positive integers, and assume that $s \geq 4$. A finite graph $\Gamma$ of valency $k$ is vertex-biprimitive and $s$-transitive if and only if either

(i) $\Gamma$ is the standard double cover of a vertex-primitive s-transitive graph (classified in Theorem 1.3); or

(ii) $\Gamma \cong \Gamma(G, H, H g H)$ such that $G, \operatorname{soc}(G) \cap H, k, s$ are as listed in Table 2, where $q$ is a prime-power, and $p$ is a prime.

Further, such a graph $\Gamma$ is uniquely determined by its order $|V \Gamma|$.

Remark. The $\mathrm{M}_{12}$-graph was used to characterize the Mathieu group $\mathrm{M}_{12}$ by Weiss [36].

This result does not help to describe all $s$-transitive graphs for $s \geq 4$ as there is no reduction to the primitive and biprimitive case. The major problem remaining is to handle the quasiprimitive and bi-quasiprimitive cases (see [20]). 
It follows from these theorems that there exist no finite vertex-primitive 7transitive graphs, and that all finite vertex-biprimitive 7 -transitive graphs of valency $k$ admit $\mathrm{G}_{2}\left(3^{m}\right)$ and have $k=3^{m}+1$, for some $m$.

Wong [39] constructed the $\operatorname{PSL}(3,3) \cdot \mathbb{Z}_{2}$-graph (see Example [3.3) and proved that it is the unique vertex-primitive cubic 5 -transitive graph. Biggs and Hoare [4] constructed an infinite family of bipartite 5 -transitive cubic graphs from $\operatorname{P\Gamma L}\left(2, p^{2}\right)$ (see Example 3.6). Motivated by Wong's classification, they further conjectured that each 5-transitive cubic biprimitive graph is either one of these graphs, or is a standard double cover of the $\operatorname{PSL}(3,3) \cdot \mathbb{Z}_{2}$-graph given in Table 1 . It is an immediate consequence of Theorems 1.3 and 1.4 that this conjecture is true.

Corollary 1.5. The only vertex-biprimitive 5-transitive graphs of valency 3 are the $\operatorname{P\Gamma L}(2,9)$-graph, which is Tutte's 8-Cage, and the standard double cover of the $\operatorname{PSL}(3,3) . \mathbb{Z}_{2}$-graph.

In Section 2, we describe generalized polygon graphs in terms of coset graphs, and state the results of Weiss 33 and Gardiner [10], which provide a method for constructing $s$-transitive graphs for $s \geq 4$. Then in Section 3 , we reproduce most of the known $s$-transitive graphs for $s \in\{4,5,7\}$ and construct some new 4-transitive graphs in a uniform manner. Finally, we complete the proofs of Theorems 1.11 .4 in Section 4 .

\section{Amalgams of $s$-TRAnsitive GRAPHS FOR $s \in\{4,5,7\}$}

In this section, we study a method of constructing $s$-transitive graphs in terms of coset graphs. Let $G$ be a finite group, and let $H$ be a subgroup of $G$. The subgroup $H$ is said to be core-free if $H$ contains no nontrivial normal subgroups of $G$. Denote by $[G: H]$ the set of right cosets of $H$ in $G$, and for a subset $S$ of $G$, denote by $\underline{S}$ the set of right cosets of $H$ contained in $H S$, that is, $\{H s \mid s \in S\}$. Recall that for an element $g \in G \backslash H$ such that $g^{2} \in H$, the coset graph $\Gamma(G, H, H g H)$ is the graph with vertex set $[G: H]$ and with $H x, H y$ adjacent if and only if $y x^{-1} \in H g H$. By definition, the set of vertices $H x$ of $\Gamma(G, H, H g H)$ which are adjacent to the vertex $H$ is equal to $\underline{g H}=\{H g h \mid h \in H\}$, denoted by $\Gamma(H)$ as usual. It follows that the group $G$ in its coset action by right multiplication on $[G: H]$ is transitive on the vertex set of $\Gamma(G, H, H g H)$, and in this $G$-action the subgroup $H$ is the stabilizer of the vertex $H$ in $G$ and acts transitively on $\Gamma(H)$. Thus $G$ acts transitively on the vertex set and the arc set of $\Gamma(G, H, H g H)$, that is, $\Gamma(G, H, H g H)$ is a $G$-arctransitive graph. The valency of $\Gamma(G, H, H g H)$ equals the cardinality of $\Gamma(H)$, that is, $|[H g H: H]|$. Clearly, $|[H g H: H]|=\left|\left[H: H \cap H^{g}\right]\right|$, and by definition, it is also clear that $\Gamma(G, H, H g H)$ is connected if and only if $\langle H, g\rangle=G$.

Let $\alpha$ and $\beta$ denote the vertex $H$ and the vertex $H g$, respectively. Then, as observed before, $G_{\alpha}=H$, and further, $G_{\beta}=H^{g}$. Since $g^{2} \in H, g$ interchanges $\alpha$ and $\beta$, and so interchanges $G_{\alpha}$ and $G_{\beta}$. Now $G_{\alpha \beta}$, the subgroup of $G$ which fixes both $\alpha$ and $\beta$, equals $H \cap H^{g}$; while $G_{\{\alpha, \beta\}}$, the subgroup of $G$ which fixes the edge $\{\alpha, \beta\}$, equals $\left\langle H \cap H^{g}, g\right\rangle$.

As we have noticed, the subgroup $H$ acts transitively on $\Gamma(H)$ and has pointstabilizer $H \cap H^{g}$. Thus the $H$-action on $\Gamma(H)$ is equivalent to the coset action of $H$ on $\left[H: H \cap H^{g}\right]$. For convenience, we summarize the above observations in the following lemma (see [27]). 
Lemma 2.1. Let $G$ be a finite group, and let $\Gamma=\Gamma(G, H, H g H)$ be a coset graph of $G$. Denote by $\alpha, \beta$ the vertices $H, H g$, respectively, of $\Gamma$. Then the following are true:

(1) $\Gamma$ is $G$-arc-transitive and has valency $\left|\left[H: H \cap H^{g}\right]\right|$;

(2) $\Gamma$ is connected if and only if $\langle H, g\rangle=G$;

(3) $G_{\alpha}=H, G_{\beta}=H^{g}, G_{\alpha \beta}=H \cap H^{g}$ and $G_{\{\alpha, \beta\}}=\left\langle H \cap H^{g}, g\right\rangle$.

These simple properties suggest some method for constructing arc-transitive graphs with certain extra conditions.

Typical examples of $s$-transitive graphs for $s \geq 4$ come from the parabolic geometries of the rank 2 Lie type groups $\mathrm{A}_{2}(q) \cong \operatorname{PSL}(3, q), \mathrm{B}_{2}\left(2^{m}\right) \cong \operatorname{Sp}\left(4,2^{m}\right)$ and $\mathrm{G}_{2}\left(3^{m}\right)$, corresponding to $s=4,5$ and 7 , respectively. These graphs are the socalled classical generalized polygon graphs (see [31] or [13, p. 423]). In this section, we first describe these graphs in terms of coset graphs, and then state a result of Weiss about automorphism groups of $s$-transitive graphs, that is the classification of the amalgams for $s$-transitive graphs for $s \in\{4,5,7\}$. Recall that for a positive integer $n,[n]$ denotes a group of order $n$.

A 4-transitive graph from $\operatorname{PSL}(3, q)$. Let $T=\operatorname{PSL}(3, q)$, where $q=p^{m}, p$ is a prime and $m \geq 1$. Then $T$ has two conjugacy classes of maximal parabolic subgroups, and has a graph automorphism $g$, which is of order 2 ; further, $g$ fuses the two conjugacy classes of maximal parabolic subgroups. Let $P$ be a maximal parabolic subgroup of $T$. Then $P=\left[q^{2}\right] \rtimes\left(\mathbb{Z}_{\frac{q-1}{(q-1,3)}}\right.$. PGL $\left.(2, q)\right)$ and $P \cap P^{g}=$ $\left[q^{3}\right] \rtimes\left(\mathbb{Z}_{q-1} \times \mathbb{Z}_{\frac{q-1}{(q-1,3)}}\right)$. Now $g^{2}=1 \in P,\langle P, g\rangle=\langle T, g\rangle$, and $\left|\left[P: P \cap P^{g}\right]\right|=q+1$. Thus the coset graph

$$
\Gamma=\Gamma(\langle T, g\rangle, P, P g P)
$$

is a bipartite $(\langle T, g\rangle, 4)$-transitive graph of valency $q+1$ (see Theorem 2.2). Moreover, Aut $\Gamma=\operatorname{Aut}(\operatorname{PSL}(3, q))$, and thus $\Gamma$ is $(G, 4)$-transitive for each $G$ with $\langle T, g\rangle \leq G \leq \operatorname{Aut}(\operatorname{PSL}(3, q))$; in particular, $\Gamma$ is 4 -transitive. Let $d, f$ be a diagonal or a field automorphism, respectively, of $T$. Then $o(d)=(q-1,3), o(f)=m$, and $d, f, P$ can be chosen so that $d, f$ normalize $P$. Let $R$ be a subgroup of $\langle d, f\rangle$, and let

$$
G=T \cdot R \cdot\langle g\rangle .
$$

Then $R$ normalizes $T, P$ and $P \cap P^{g}$. Thus $T . R$ has a subgroup $H:=P . R$ such that $L:=H \cap H^{g}=\left(P \cap P^{g}\right) . R$. Then we have

$$
H=\left(\left[q^{2}\right] \rtimes\left(\mathbb{Z}_{\frac{q-1}{(q-1,3)}} \cdot \operatorname{PGL}(2, q)\right)\right) \cdot R, \quad L=\left(\left[q^{3}\right] \rtimes\left(\mathbb{Z}_{q-1} \times \mathbb{Z}_{\frac{q-1}{(q-1,3)}}\right)\right) \cdot R .
$$

Finally, we note that $\mathbf{N}_{G}(L)=L \cdot \mathbb{Z}_{2}$ (see [16]).

A 5 -transitive graph from $\operatorname{Sp}\left(4,2^{m}\right)$. Let $T=\operatorname{Sp}(4, q)$, where $q=2^{m}$ for some $m \geq 1$. Then $T$ has two conjugacy classes of maximal parabolic subgroups, which are fused in $\operatorname{Aut}(T)$. Let $f$ be a field automorphism, and let $g \in \operatorname{Aut}(T) \backslash \operatorname{Inn}(T) .\langle f\rangle$. (Note that we are identifying $T$ with $\operatorname{Inn}(T)$.) Then $o(f) \mid m$, Aut $(T) /\langle T, f\rangle \cong \mathbb{Z}_{2}$ and $g^{2} \in\langle f\rangle$. Let $P$ be a maximal parabolic subgroup of $T$. Then $g, f, P$ may be chosen so that $f$ normalizes $P$ but $g$ does not, and $P=\left[q^{3}\right] \rtimes \mathrm{GL}(2, q)$. Let $R \leq\langle f\rangle$ contain $g^{2}$, and set

$$
G=T \cdot\langle R, g\rangle
$$


TABLE 3 .

\begin{tabular}{|c|c|c|c|}
\hline$k=q+1$ & $s$ & $G_{\alpha}=H$ & $G_{\alpha \beta}=L$ \\
\hline \hline$q+1$ & 4 & $\left(\left[q^{2}\right] \rtimes\left(\mathbb{Z}_{\frac{q-1}{(q-1,3)}} \cdot \operatorname{PGL}(2, q)\right)\right) \cdot R$ & $\left(\left[q^{3}\right] \rtimes\left(\mathbb{Z}_{q-1} \times \mathbb{Z}_{\frac{q-1}{(q-1,3)}}\right) \cdot R\right.$ \\
\hline $2^{m}+1$ & 5 & $\left(\left[q^{3}\right] \rtimes \mathrm{GL}(2, q)\right) \cdot R$ & $\left(\left[q^{4}\right] \rtimes \mathbb{Z}_{q-1}^{2}\right) \cdot R$ \\
\hline $3^{m}+1$ & 7 & $\left(\left[q^{5}\right] \rtimes \mathrm{GL}(2, q)\right) \cdot R$ & $\left(\left[q^{6}\right] \rtimes \mathbb{Z}_{q-1}^{2}\right) \cdot R$ \\
\hline
\end{tabular}

Then T.R has a subgroup $H:=P . R$ such that $g^{2} \in H$ and $\langle H, g\rangle=G$. Thus the coset graph $\Gamma=\Gamma(G, H, H g H)$ is a $(G, 5)$-transitive graph of valency $q+1$ (see Theorem [2.2). Moreover, Aut $\Gamma=\operatorname{Aut}(T)$, and $\Gamma$ is 5 -transitive. The subgroups $H, L<G$ have the form

$$
H=\left(\left[q^{3}\right] \rtimes \mathrm{GL}(2, q)\right) \cdot R, \quad L=\left(\left[q^{4}\right] \rtimes \mathbb{Z}_{q-1}^{2}\right) \cdot R .
$$

We notice that $\mathbf{N}_{G}(L)=L \cdot \mathbb{Z}_{2}$ (see [16]).

A 7-transitive graph from $\mathrm{G}_{2}\left(3^{m}\right)$. Let $T=\mathrm{G}_{2}(q)$, where $q=3^{m}$ for some $m \geq 1$. Then $T$ has two conjugacy classes of maximal parabolic subgroups, and has a graph automorphism $g$, which is of order 2; further, $g$ fuses the two conjugacy classes of parabolic subgroups. Let $P$ be a parabolic subgroup of $T$. Then $P=$ $\left[q^{5}\right] \rtimes \mathrm{GL}(2, q)$, and $P, g$ may be chosen so that $P \cap P^{g}=\left[q^{6}\right] \rtimes \mathbb{Z}_{q-1}^{2}$. Now $g^{2}=1 \in P,\langle P, g\rangle=\langle T, g\rangle$, and $\left|\left[P: P \cap P^{g}\right]\right|=q+1$. Thus the coset graph

$$
\Gamma=\Gamma(\langle T, g\rangle, P, P g P)
$$

is a bipartite $(\langle T, g\rangle, 7)$-transitive graph of valency $q+1$ (see Theorem 2.2). Moreover, Aut $\Gamma=\operatorname{Aut}\left(\mathrm{G}_{2}(q)\right)$, and thus $\Gamma$ is $(G, 7)$-transitive for each $G$ with $\langle T, g\rangle \leq$ $G \leq \operatorname{Aut}\left(\mathrm{G}_{2}(q)\right)$; in particular, $\Gamma$ is 7 -transitive. Choose a field automorphism $f$ of $T$ such that $f$ normalizes $P$. Let $R$ be a subgroup of $\langle f\rangle$, and let

$$
G=T \cdot R \cdot\langle g\rangle \text {. }
$$

Then $R$ normalizes $T, P$ and $P \cap P^{g}$. Thus $T . R$ has a subgroup $H:=P . R$ such that $L:=H \cap H^{g}=\left(P \cap P^{g}\right)$. R. Then we have

$$
H=\left(\left[q^{5}\right] \rtimes \mathrm{GL}(2, q)\right) \cdot R, \quad L=\left(\left[q^{6}\right] \rtimes \mathbb{Z}_{q-1}^{2}\right) \cdot R .
$$

Finally, we note that, by [15, Theorem B], $\mathbf{N}_{G}(L)=L . \mathbb{Z}_{2}$.

These three classes of graphs are important because of the following result (see [10] and [33, 35], also refer to [13, Theorem 2.1.5]).

Theorem 2.2. Let $\Gamma$ be a connected graph of valency $k$, and let $G \leq$ Aut $\Gamma$ be such that $\Gamma$ is transitive on $V \Gamma$. Let $\alpha, \beta \in V \Gamma$ be a pair of adjacent vertices.

(1) (Weiss [33, 35]) Assume that $s \geq 4$ and that $\Gamma$ is $(G, s)$-transitive. Then $s \in\{4,5,7\}, k=q+1$ where $q$ is a prime power, and the stabilizers $G_{\alpha}$ and $G_{\alpha \beta}$ are completely known, as in Table 3 . Further, either $\Gamma$ is s-transitive, or $k=3, s=4$, and $\Gamma$ is 5-transitive.

(2) (Gardiner [10, Theorem 3.1]) Assume that $H$ is a subgroup of $G$ which satisfies line 1,2 or 3 of Table 3 . If there exists an arc-transitive graph $\Gamma(G, H, H g H)$ of valency $k$, then $\Gamma(G, H, H g H)$ is s-transitive for $s=4,5$ or 7 , respectively.

Remark on Theorem [2.2. The valency of the graph $\Gamma$ and the arc-stabilizer $L$ are uniquely determined by the point-stabilizer $H$. Actually, $L$ is the normalizer of a Sylow $p$-subgroup of $H$, where $q$ is a $p$-power, and the valency is equal to $|H| /|L|$. 
Hence, in particular, all subgroups of $H$ isomorphic to $L$ are conjugate (in $H$ ) to $L$.

To end this section, we describe a possible (and often efficient) method for constructing $s$-arc transitive graphs for $s \geq 4$. Let $\Gamma(G, H, H g H)$ be a coset graph, and let $L=H \cap H^{g}$ and $K=\langle L, g\rangle$. Then we have three subgroups $H, L, K$ of $G$, which have the properties:

$H \cap K=L,|[K: L]|=2$, and $|[H: L]|$ equals the valency of $\Gamma(G, H, H g H)$.

A triple $(H, L, K)$ with these properties is called an amalgam (see [11, 13). Thus, constructing arc-transitive graphs with certain extra property is equivalent to seeking suitable groups $G$ and suitable pairs $\{H, g\}$; the latter is in turn equivalent to seeking suitable amalgams $(H, L, K)$. A procedure for constructing $s$-transitive graphs may be stated as follows:

(A) find a suitable group $G$ which has a subgroup $H$ as listed in Table 3;

(B) find a subgroup $L$ of $H$ such that $H$ acting on $[H: L]$ is of degree $q+1$;

(C) analyze $\mathbf{N}_{G}(L)$ to decide whether there exists a 2-element $g \in \mathbf{N}_{G}(L) \backslash L$ such that $g^{2} \in H$ and $\langle H, g\rangle=G$.

We note that there may be different 2-elements $g \in \mathbf{N}_{G}(L) \backslash L$ such that $g^{2} \in H$ and $\langle H, g\rangle=G$. Thus for a pair $(G, H)$, there may be different arc-transitive graphs $\Gamma(G, H, H g H)$, which in general are not isomorphic. However, for certain $s$ transitive graphs for $s \geq 4$, we have the uniqueness of such graphs as in Lemma 2.3.

Lemma 2.3. Let $\Gamma=\Gamma(G, H, H g H)$ be a connected $(G, s)$-transitive graph, where $s \in\{4,5,7\}$, and let $L=H \cap H^{g}$. Assume that $\mathbf{N}_{G}(L)=K=L . \mathbb{Z}_{2}$. For any 2-element $f \in G$ with $f^{2} \in H$, if $\Gamma(G, H, H f H)$ is connected and s-transitive, then

$$
\Gamma(G, H, H f H)=\Gamma(G, H, H g H) .
$$

Proof. By Theorem [2.2, $H \cap H^{f} \cong L$. It then follows from the remark on Theorem 2.2 that $H \cap H^{f}$ is conjugate in $H$ to $L$. Hence

$$
H \cap H^{h^{-1} f h}=\left(H \cap H^{f}\right)^{h}=L
$$

for some $h \in H$. Since $\left(h^{-1} f h\right)^{2} \in H$, we have that

$$
L^{h^{-1} f h}=\left(H \cap H^{h^{-1} f h}\right)^{h^{-1} f h}=H^{h^{-1} f h} \cap H=L,
$$

that is, $h^{-1} f h \in \mathbf{N}_{G}(L)$. Since $\left|\mathbf{N}_{G}(L) / L\right|=2$ and $g \in \mathbf{N}_{G}(L) \backslash L$, we have $\mathbf{N}_{G}(L)=L \cup L g$. Hence $h^{-1} f h \in \mathbf{N}_{G}(L) \backslash L=L g$, and so $h^{-1} f h=h^{\prime} g$ for some $h^{\prime} \in L$. Therefore, $H f H=H h^{-1} f h H=H h^{\prime} g H=H g H$, and thus we have that $\Gamma(G, H, H f H)=\Gamma(G, H, H g H)$.

An immediate consequence of this lemma is

Corollary 2.4. The classical generalized polygon graphs (constructed above) are uniquely determined by their automorphism groups.

\section{Constructing $s$-ARC transitive GraphS}

In this section, we will first describe the constructions of most known examples of $s$-arc transitive graphs for $s \in\{4,5,7\}$ in a uniform way, apart from the polygonal graphs described in Section 2, and then construct some new 4-transitive graphs: one from the Monster simple group M; an infinite family of graphs from $\operatorname{PSp}(4, p)$ with $p$ a prime. (As usual, for positive integers $m$ and $n$, by the notation $m \mid n$ we 
mean $m$ divides $n$, and by $m \| n$ we mean $m$ strictly divides $n$, that is, $m \mid n$ and $m$ is coprime to $n / m$.)

In the following, for a finite group $G$ and a prime $p|| G \mid$, denote by $G_{p}$ a Sylow $p$-subgroup of $G$. The first lemma provides a method to construct certain arctransitive graphs.

Lemma 3.1. Let $G$ be a finite group. Assume that $G$ has a maximal subgroup $H$ which is core-free, and assume further that $H$ contains a subgroup $P$ such that $\left|\mathbf{N}_{G}(P)\right| /\left|\mathbf{N}_{H}(P)\right|$ is even. Then there exists $g \in G \backslash H$ such that $\Gamma:=$ $\Gamma(G, H, H g H)$ is a connected $G$-arc transitive graph of valency $\left|\left[H: H \cap H^{g}\right]\right|$.

Proof. By the assumption, there exists a 2-element $g \in \mathbf{N}_{G}(P) \backslash \mathbf{N}_{H}(P)$ such that $g^{2} \in \mathbf{N}_{H}(P)$. Thus we have $g^{2} \in H$, and $g \notin H$. Since $H$ is a maximal subgroup of $G, G=\langle H, g\rangle$. By Lemma 2.1 1$)$, the coset graph $\Gamma(G, H, H g H)$ is a connected $G$-arc transitive graph of valency $\left|\left[H: H \cap H^{g}\right]\right|$.

Lemma 3.2. Let $G$ be a finite group, and let $K$ be a 2-subgroup of $G$. Assume that $|G| /|K|$ is even. Then $\mathbf{N}_{G}(K) / K$ is of even order.

Proof. Let $G_{2}$ be a Sylow 2-subgroup of $G$ which contains $K$. Then $G_{2}>K$, and by [30, p. 88], $\mathbf{N}_{G_{2}}(K)>K$, so the conclusion of the lemma follows.

In the rest of this section, we describe some constructions of $s$-transitive graphs for $s \in\{4,5,7\}$. All of them involve nonabelian simple groups.

3.1. Graphs of valency 3 and 4 . Note that $\mathrm{GL}(2,2) \cong \mathrm{S}_{3}, \mathbb{Z}_{2}^{2} \rtimes \mathrm{GL}(2,2) \cong \mathrm{S}_{4}$, and $\mathbb{Z}_{2}^{3} \rtimes \mathrm{GL}(2,2) \cong \mathrm{S}_{4} \times \mathbb{Z}_{2}$. Thus, for cubic $s$-transitive graphs, the amalgams have been often written as special forms, that is, either

$$
\begin{aligned}
& s=4 \text { and }(H, L)=\left(\mathrm{S}_{4}, \mathrm{D}_{8}\right), \text { or } \\
& s=5 \text { and }(H, L)=\left(\mathrm{S}_{4} \times \mathbb{Z}_{2}, \mathrm{D}_{8} \times \mathbb{Z}_{2}\right) .
\end{aligned}
$$

In particular, in either case $L$ is a 2 -group. Assume that $G$ is a finite group which has subgroups $H$ and $L$ satisfying these conditions. By Lemma 3.2, if $|G| /|L|$ is even, then $\mathbf{N}_{G}(L) / L$ is of even order, and thus there exists a 2-element $g \in \mathbf{N}_{G}(L) \backslash H$ such that $g^{2} \in H$. Assume further that $H$ is a maximal subgroup. Then $\langle H, g\rangle=G$. Therefore, we have the following examples of $s$-transitive graphs for $s=4$ or 5 . These were first constructed by Wong (1967).

Example 3.3. Let

$$
G=\operatorname{Aut}(\operatorname{PSL}(3,3))=\operatorname{PSL}(3,3) \cdot \mathbb{Z}_{2} .
$$

By the Atlas [8], $G$ has a maximal subgroup

$$
H \cong \mathrm{S}_{4} \times \mathbb{Z}_{2},
$$

and $|G| /|H|$ is even. Thus there exists a vertex-primitive $(G, 5)$-transitive cubic graph $\Gamma=\Gamma(G, H, H g H)$ such that Aut $\Gamma=G$ and

$$
L=H \cap H^{g} \cong \mathrm{D}_{8} \times \mathbb{Z}_{2} .
$$

Noting that $2^{5} \||G|$ (see the Atlas [8]), it follows from Lemma 3.2 that $\mathbf{N}_{G}(L)=$ $\left[2^{5}\right]$, and so $\mathbf{N}_{G}(L) / L \cong \mathbb{Z}_{2}$. By Lemma 2.3, $\Gamma$ is a unique $(G, 5)$-transitive cubic graph. 
Example 3.4. Let

$$
G=\operatorname{PSL}(2, p) \text { where } p \equiv \pm 1(\bmod 16) \text { is a prime. }
$$

Then by [30, p. 417], $G$ has a maximal subgroup

$$
H \cong \mathrm{S}_{4},
$$

and thus $|G| / \mid H$ is even. So there exists a vertex-primitive $(G, 4)$-transitive cubic graph $\Gamma=\Gamma(G, H, H g H)$ such that Aut $\Gamma=G$ and

$$
L=H \cap H^{g} \cong \mathrm{D}_{8} .
$$

Checking subgroups of $G$ which contain $L$ (see [30, p. 417]), we conclude that $\mathbf{N}_{G}(L) \cong \mathrm{D}_{16}$ and so $\mathbf{N}_{G}(L) / L \cong \mathbb{Z}_{2}$. By Lemma 2.3 , $\Gamma$ is a unique $(G, 4)$-transitive cubic graph.

Assume now that $|G| /|L|$ is odd but $|\operatorname{Aut}(G)| /|L|$ is even. Then in some circumstances there exists a 2-element $g \in \mathbf{N}_{\langle G, g\rangle}(L) \backslash H$ such that $g^{2} \in H$. If, in addition, $H$ is a maximal subgroup of $G$, then $\langle H, g\rangle=\langle G, g\rangle$. Thus we have the next examples of bipartite $s$-transitive graphs for $s=4$ or 5 . These were first constructed by Biggs and Hoare [4] (also see [3, 18b]).

Example 3.5. Let $T=\operatorname{PSL}(2, p)$ where $p \equiv \pm 1(\bmod 8)$. Then $T$ has two conjugacy classes of maximal subgroups $\mathrm{S}_{4}$, which are fused in

$$
G=\operatorname{PGL}(2, p),
$$

see [30 p. 417]. Let $H$ be such that

$$
\mathrm{S}_{4} \cong H<T .
$$

Then $|T| /|H|$ is odd but $|G| /|H|$ is even. Thus there exists a 2-element $g \in G \backslash H$ such that $g^{2} \in H,\langle H, g\rangle=G$ and $H \cap H^{g}=L$. Thus there exists a bipartite $(G, 4)$ transitive cubic graph $\Gamma=\Gamma(G, H, H g H)$ such that Aut $\Gamma=G . \mathbb{Z}_{2}=\operatorname{PGL}(2, p)$ and

$$
L=H \cap H^{g} \cong \mathrm{D}_{8} .
$$

Checking subgroups of $G$ which contain $L$ (see [30, p. 417]), we may conclude that $\mathbf{N}_{G}(L)$ is a Sylow 2 -subgroup of $G$, which is of order 16 . Thus $\mathbf{N}_{G}(L) / L \cong \mathbb{Z}_{2}$. By Lemma 2.3, $\Gamma$ is a unique $(G, 4)$-transitive cubic graph.

The 5-transitive cubic graphs given in the next example were also constructed by Biggs and Hoare [4].

Example 3.6. Let $T=\operatorname{PSL}\left(2, p^{2}\right)$ where $p$ is a prime and $p^{2} \equiv \pm 1(\bmod 8)$. Then $\operatorname{Out}(T) \cong \mathbb{Z}_{2}^{2}$ and

$$
G:=\operatorname{Aut}(T)=\operatorname{P\Gamma L}\left(2, p^{2}\right) .
$$

By [30, p. 417], $T$ has two conjugacy classes of subgroups which are isomorphic to $\mathrm{S}_{4}$, which are fused in $G$. It follows that the group $\mathrm{P} \Sigma \mathrm{L}\left(2, p^{2}\right)$ has two conjugacy classes of subgroups that are isomorphic to $\mathrm{S}_{4} \times \mathbb{Z}_{2}$, which are fused in $G$. Let $H$ be a subgroup of $\mathrm{P} \Sigma \mathrm{L}\left(2, p^{2}\right)$ such that

$$
H \cong \mathrm{S}_{4} \times \mathbb{Z}_{2} .
$$


Then a Sylow 2-subgroup $L$ of $H$ is isomorphic to $\mathrm{D}_{8} \times \mathbb{Z}_{2}$, and is a proper subgroup of a Sylow 2-subgroup $S$ of $G$. Therefore, there exists a 2-element $g \in S \backslash L$ normalizing $L$, and so

$$
H \cap H^{g}=L \cong \mathrm{D}_{8} \times \mathbb{Z}_{2} .
$$

Now it is easily shown that $\langle H, g\rangle$ is contained in no proper subgroup of $G$, so $\langle H, g\rangle=G$. Therefore, by Lemma 3.1 and Theorem 2.2, the coset graph $\Gamma=$ $\Gamma(G, H, H g H)$ is a 5-transitive cubic graph, which is bipartite. Analyzing subgroups of $G$ which contain $L$ (see [30, p. 417]), we may obtain that $\mathbf{N}_{G}(L)$ is a Sylow 2subgroup of $G$, which has order 32 . Hence $\mathbf{N}_{G}(L) / L \cong \mathbb{Z}_{2}$. By Lemma 2.3, $\Gamma$ is a unique $(G, 5)$-transitive cubic graph. Since $H$ is maximal in $\mathrm{P} \Sigma \mathrm{L}\left(2, p^{2}\right)$ if and only if $p=3, \Gamma$ is biprimitive if and only if $p=3$. We note that $\operatorname{PSL}(2,9) \cong \mathrm{A}_{6}$, and the corresponding graph is Tutte's 8-Cage.

Next we consider 4-valent graphs. Note that $\mathrm{GL}(2,3) \cong \mathrm{Q}_{8} \rtimes \mathrm{S}_{3}$. Thus, for $s$-transitive graphs of valency 4 with $s \in\{4,5,7\}$, the amalgams also have a special forms, that is, either

$$
s=4 \text { and }(H, L)=\left(\mathbb{Z}_{3}^{2} \rtimes\left(\mathrm{Q}_{8} \rtimes \mathrm{S}_{3}\right),\left[3^{3}\right] \rtimes \mathbb{Z}_{2}^{2}\right),
$$

or

$$
s=7 \text { and }(H, L)=\left(\left[3^{5}\right] \rtimes\left(\mathrm{Q}_{8} \rtimes \mathrm{S}_{3}\right),\left[3^{6}\right] \rtimes \mathbb{Z}_{2}^{2}\right) .
$$

We note that in this case $L=\mathbf{N}_{H}\left(L_{3}\right)$, where $L_{3}$ is a Sylow 3-subgroup of $H$, and thus $\mathbf{N}_{G}(L) \leq \mathbf{N}_{G}\left(L_{3}\right)$. The 4-transitive graph given in the next lemma was constructed and used to characterize the group $\mathrm{M}_{12}$ by Weiss [36].

Lemma 3.7. Let $T=\mathrm{M}_{12}$, the Mathieu simple group of degree 12 , and let $G=$ $\operatorname{Aut}(T)=T \cdot \mathbb{Z}_{2}$. Then there exists a unique connected 4 -transitive graph $\Gamma$ of valency 4 such that $\Gamma$ is biprimitive and Aut $\Gamma=G$.

Proof. By the Atlas 8], $T$ has two conjugacy classes of maximal subgroups isomorphic to $\mathbb{Z}_{3}^{2} \rtimes\left(\mathbb{Z}_{2}\right.$. $\left.\mathrm{S}_{4}\right)$, which are fused in $G$ into maximal subgroups of $G$ isomorphic to $3_{+}^{1+2} \rtimes \mathrm{D}_{8}$. Let $H$ be a subgroup of $T$ such that

$$
H \cong \mathbb{Z}_{3}^{2} \rtimes\left(\mathbb{Z}_{2} \cdot \mathrm{S}_{4}\right)
$$

(We note that in this case, $H$ is isomorphic to $\mathbb{Z}_{3}^{2} \rtimes \mathrm{GL}(2,3)$.) Let $H_{3}$ be a Sylow 3 -subgroup of $H$ and let $L=\mathbf{N}_{H}\left(H_{3}\right)$. Then $H_{3} \cong 3_{+}^{1+4}$, and

$$
L \cong 3_{+}^{1+4} \rtimes\left[2^{2}\right] .
$$

It follows from the Atlas [8] that $\mathbf{N}_{G}(L)=3_{+}^{1+4} \rtimes \mathrm{D}_{8}$. Thus there exists a 2-element $g \in \mathbf{N}_{G}(L) \backslash H$ such that $L=H \cap H^{g}$. Since $H$ is a maximal subgroup of $T$, it follows that $\langle H, g\rangle=G$. Thus, by Lemma 2.1, the coset graph $\Gamma=\Gamma(G, H, H g H)$ is a connected arc-transitive graph of valency 4, and further by Lemma 3.1 and Theorem 2.2, $\Gamma$ is 4-transitive. Since $g \notin T, T$ is not transitive on $V \Gamma$, and thus $\Gamma$ is bipartite, say with parts $\Delta_{1}$ and $\Delta_{2}$. As $H$ is a maximal subgroup of $T, \Gamma$ is biprimitive. It follows from Theorem 2.2 that Aut $\Gamma=G$. Since $\mathbf{N}_{G}(L) / L \cong \mathbb{Z}_{2}$, by Lemma 2.3. $\Gamma$ is the unique $(G, 4)$-transitive graph of valency 4 . 
An infinite family of non-bipartite 5-transitive cubic graphs was constructed by Conder (1988) from the alternating groups $\mathrm{A}_{n}$ and the symmetric groups $\mathrm{S}_{n}$. Very recently, an infinite family of non-bipartite 7-transitive graphs of valency 4 was constructed by Conder and Walker (1998), also from the alternating groups $\mathrm{A}_{n}$ and the symmetric groups $\mathrm{S}_{n}$. These graphs are neither vertex-primitive nor vertex-biprimitive.

3.2. Graphs of valency 5. Weiss (1986) constructed a 4-transitive graph of valency 5 from the Janko simple group $\mathrm{J}_{3}$, and used the graph to characterize the simple group. Here we first give a description for the Weiss graph, and then construct an infinite family of 4-transitive graphs of valency 5 from a class of symplectic simple groups. We notice that for a $(G, 4)$-transitive graph of valency 5 ,

$$
\mathbb{Z}_{2}^{4} \rtimes \mathrm{GL}(2,4) \leq H \leq\left(\mathbb{Z}_{2}^{4} \rtimes \mathrm{GL}(2,4)\right) \cdot \mathbb{Z}_{2},
$$

and

$$
\left[2^{6}\right] \cdot \mathbb{Z}_{3}^{2} \leq L \leq\left[2^{6}\right] \cdot \mathbb{Z}_{3}^{2} \cdot \mathbb{Z}_{2}
$$

in particular, $L$ is a Hall $\{2,3\}$-subgroup of $H$.

Lemma 3.8. Let $T$ be the Janko simple group $\mathrm{J}_{3}$. Then there exists a unique 4-transitive graph $\Gamma$ of valency 5 such that Aut $\Gamma=\operatorname{Aut}(T)=T . \mathbb{Z}_{2}$ and $\Gamma$ is vertex-primitive.

Proof. Let $G=\operatorname{Aut}(T)$. By the Atlas [8], $G$ has one conjugacy class of maximal subgroups isomorphic to $\mathbb{Z}_{2}^{4} \rtimes\left(\mathbb{Z}_{3} \times A_{5}\right) . \mathbb{Z}_{2} \cong \mathbb{Z}_{2}^{4} \rtimes \mathrm{GL}(2,4) \cdot \mathbb{Z}_{2}$. Let $H<G$ be such that

$$
H \cong \mathbb{Z}_{2}^{4} \rtimes \mathrm{GL}(2,4) \cdot \mathbb{Z}_{2}
$$

and let $L$ be a Hall $\{2,3\}$-subgroup of $H$. Then

$$
L \cong\left[2^{6}\right] \cdot \mathbb{Z}_{3}^{2} \cdot \mathbb{Z}_{2}
$$

and by the Atlas 8, $\mathbf{N}_{G}(L)=2^{2+4} \rtimes\left(\mathrm{S}_{3} \times \mathrm{S}_{3}\right)=L . \mathbb{Z}_{2}$. Thus there exists a 2element $g$ in $\mathbf{N}_{G}(L) \backslash H$ such that $\langle H, g\rangle=G, g^{2} \in H, L=H \cap H^{g}$ and $|H: L|=5$. Therefore, by Lemma 2.1 and Theorem 2.2, the coset graph $\Gamma=\Gamma(G, H, H g H)$ is a 4-transitive graph of valency 5 , which is the Weiss graph given in [37]. It follows from Theorem 2.2 that Aut $\Gamma=G$. Finally, by Lemma 2.3, $\Gamma$ is a unique $(G, 4)$ transitive graph of valency 5 .

Next we construct an infinite family of 4-transitive graphs of valency 5 .

Lemma 3.9. Let $G=\operatorname{PSp}(4, p)$, where $p$ is a prime and $2^{4} .3 \|\left(p^{2}-1\right)$. Then there exists a unique 4-transitive graph $\Gamma$ of valency 5 such that $G_{\alpha} \cong \mathbb{Z}_{2}^{4} \rtimes \mathrm{S}_{5}$, Aut $\Gamma=G$ and $\Gamma$ is vertex-primitive.

Proof. By [16, Proposition 4.6.9], $G$ has one conjugacy class of maximal subgroups isomorphic to $\mathbb{Z}_{2}^{4} \cdot\left(\Omega_{4}^{-}(2) \cdot \mathbb{Z}_{2}\right) \cong \mathbb{Z}_{2}^{4} \cdot \operatorname{PGL}(2,4) \cong \mathbb{Z}_{2}^{4} \cdot \mathrm{S}_{5}$. Let $H$ be a maximal subgroup of $G$ such that

$$
H \cong \mathbb{Z}_{2}^{4} \cdot \mathrm{S}_{5},
$$

and let $L$ be a subgroup of $H$ such that

$$
L \cong \mathbb{Z}_{2}^{4} \cdot \mathrm{S}_{4}
$$


Write $H=N_{1} \cdot \mathrm{S}_{5}$, and $L=N_{1} \cdot\left(N_{2} \cdot \mathrm{S}_{3}\right)$, where $N_{1} \cong \mathbb{Z}_{2}^{4}$ and $N_{2} \cong \mathbb{Z}_{2}^{2}$. Let $L_{2}$ be a Sylow 2-subgroup of $L$, and let $G_{2}$ be a Sylow 2-subgroup of $G$ containing $L_{2}$. Then $\left|G_{2}\right|=2^{8},\left|L_{2}\right|=2^{7}$, and so $G_{2}>L_{2}$. Now $M:=\mathbf{O}_{2}(L)=N_{1} \cdot N_{2}$. Since $\mathbf{C}_{H}\left(N_{1}\right)=N_{1}$, we have that $M$ is not abelian, and hence $M$ has exponent 4 . Let $b \in M$ be of order 4 . Then $\bar{b}=b N_{1} \in\left(N_{1} . \mathrm{A}_{4}\right) / N_{1}$ is an involution. It is clear that each involution of $\mathrm{A}_{4}$ is a square of an element of $\mathrm{S}_{4}$ of order 4 , that is, there exists $\bar{c} \in L / N_{1}=\mathrm{S}_{4}$ such that $\bar{c}^{2}=\bar{b}$. Let $c$ be a preimage of $\bar{c}$ in $L$. Then the order of $c$ is divisible by 8 , and so $L_{2}$ is of exponent 8 .

Since $G_{2}>L_{2}$, there exists $g \in \mathbf{N}_{G}\left(L_{2}\right) \backslash L_{2}$ such that $g^{2} \in L_{2}$. Suppose that $M^{g} \neq M$. Then, since $M, M^{g}$ are subgroups of $L_{2}$ of index $2,\left\langle M, M^{g}\right\rangle=M \circ M^{g}=$ $L_{2}$. However, as $M$ is of exponent 4, every element of $M \circ M^{g}$ is of order at most 4, which is a contradiction since $L_{2}=M \circ M^{g}$ is of exponent 8. Hence $M^{g}=M$, that is $g$ normalizes $M$. In particular, $M$ is a normal subgroup of $\langle L, g\rangle$. It is now easy to obtain that $\langle L, g\rangle / M$ is of order 12 and thus $g$ normalizes $L$, so that $L=H \cap H^{g}$. Hence the coset graph $\Gamma=\Gamma(G, H, H g H)$ is arc-transitive and of valency 5 . It then follows from Theorem 2.2 that $\Gamma$ is 4 -transitive and Aut $\Gamma=G$. Analyzing maximal subgroups of $G$ which contain $L$ (see [16]), we may conclude that $\mathbf{N}_{G}(L)=\langle L, g\rangle \cong L . \mathbb{Z}_{2}$. By Lemma 2.3, $\Gamma$ is a unique $(G, 4)$-transitive graph of valency 5 .

3.3. Graphs of valency greater than 5. Here we will construct two 4-transitive graphs of valency 6 , and a 4 -transitive graph of valency 14 . We observe that if $k=p+1 \geq 6$ for some prime $p$, then a $(G, 4)$-arc transitive graph of valency $k$ is 4-transitive;

$$
H=\mathbb{Z}_{p}^{2} \rtimes \mathbb{Z}_{\frac{p-1}{(p-1,3)}} \cdot \operatorname{PGL}(2, p),
$$

and

$$
L=\left[p^{3}\right] \rtimes\left(\mathbb{Z}_{p-1} \times \mathbb{Z}_{\frac{p-1}{(p-1,3)}}\right) .
$$

In particular, $L=\mathbf{N}_{H}\left(L_{p}\right)$ for a Sylow $p$-subgroup $L_{p}$. Thus $\mathbf{N}_{G}(L) \leq \mathbf{N}_{G}\left(L_{p}\right)$. The construction of the graphs of valency 6 in the next lemma was first obtained by Stroth and Weiss [28].

Lemma 3.10. Let $T$ be one of the sporadic simple groups $\mathrm{Ru}$, Th or $\mathrm{M}$. Then there exists a unique 4-transitive graph $\Gamma$ such that Aut $\Gamma=T$, and further, $\Gamma$ has valency 6 in the cases where $T=\mathrm{Ru}$ or $\mathrm{Th}$, while $\Gamma$ has valency 14 when $T=\mathrm{M}$.

Proof. Since the construction for the Ru-graph is similar to the construction for the Th-graph, we only describe the latter.

Let $T$ be the Thompson group Th. By the Atlas [8, $T$ has one conjugacy class of maximal subgroups that are isomorphic to $\mathbb{Z}_{5}^{2} \rtimes \mathbb{Z}_{4}$. $\mathrm{S}_{5} \cong \mathbb{Z}_{5}^{2} \rtimes \mathrm{GL}(2,5)$. Let $H$ be a subgroup of $T$ such that

$$
H \cong \mathbb{Z}_{5}^{2} \rtimes \mathrm{GL}(2,5),
$$

and let $H_{5}$ be a Sylow 5 -subgroup of $H$. Then

$$
L:=\mathbf{N}_{H}\left(H_{5}\right) \cong 5_{+}^{1+2} \cdot\left(\mathbb{Z}_{4} \cdot \mathbb{Z}_{4}\right),
$$

and however, by the Atlas [8], $\mathbf{N}_{T}\left(H_{5}\right) \cong 5_{+}^{1+2} \cdot\left(\mathbb{Z}_{4} \cdot \mathrm{S}_{4}\right)$. Let $M$ be a Hall $\{2,5\}$ subgroup of $\mathbf{N}_{T}\left(H_{5}\right)$ which contains $L$. Then $L$ has index 2 in $M$, and thus there exists a 2-element $g \in M \backslash L$ such that $g^{2} \in L<H$ and $g$ normalizes $L$. It follows that $L=H \cap H^{g}$. Now $|H: L|=6$, and as $H$ is a maximal subgroup of $T,\langle H, g\rangle=$ 
T. Therefore, by Lemma 3.1 and Theorem 2.2, the coset graph $\Gamma=\Gamma(T, H, H g H)$ is a connected 4 -transitive graph of valency 6 . It also follows from Theorem [2.2 that Aut $\Gamma=T$. Now $H$ has a normal subgroup $N$ such that $5_{+}^{1+2} \cdot \mathbb{Z}_{4} \cong N \triangleleft L$. Thus $N \triangleleft L \triangleleft \mathbf{N}_{T}(L) \leq \mathbf{N}_{T}\left(H_{5}\right)=N$. $\mathrm{S}_{4}$. Thus $\mathbb{Z}_{4} \cong L / N \triangleleft \mathbf{N}_{T}(L) / N \leq \mathrm{S}_{4}$, and so $\mathbf{N}_{T}(L) / N \cong \mathrm{D}_{8}$. In particular, $\mathbf{N}_{T}(L) / L \cong \mathbb{Z}_{2}$, and so by Lemma 2.3 is a unique $(T, 4)$-transitive graph of valency 6 .

Now let $T$ be the Monster simple group M. By the Atlas [8], $T$ has one conjugacy class of maximal subgroups which are isomorphic to $\mathbb{Z}_{13}^{2} \rtimes\left(\mathbb{Z}_{4}\right.$. PSL $\left.(2,13) \cdot \mathbb{Z}_{2}\right)$. Let $H$ be a subgroup of $T$ such that

$$
H \cong \mathbb{Z}_{13}^{2} \rtimes\left(\mathbb{Z}_{4} \cdot \operatorname{PSL}(2,13) \cdot \mathbb{Z}_{2}\right),
$$

and let $H_{13}$ be a Sylow 13-subgroup of $H$. Then

$$
L:=\mathbf{N}_{H}\left(H_{13}\right) \cong 13_{+}^{1+2} \cdot\left(\mathbb{Z}_{3} \times\left(\mathbb{Z}_{4} \cdot \mathbb{Z}_{4}\right)\right),
$$

and however, by the Atlas [8], $\mathbf{N}_{T}\left(H_{13}\right) \cong 13_{+}^{1+2} \rtimes\left(\mathbb{Z}_{3} \times \mathbb{Z}_{4} . \mathrm{S}_{4}\right)$. Let $L_{\{2,3\}}$ and $P$ be Hall $\{2,3\}$-subgroups of $L$ and $\mathbf{N}_{T}\left(H_{13}\right)$, respectively, such that $L_{\{2,3\}}<$ $P$. It follows that either $L_{\{2,3\}} \triangleleft P$, or $\mathbf{N}_{P}\left(L_{\{2,3\}}\right) \cong \mathbb{Z}_{3} \times\left[2^{5}\right]$. In either case, $|P| /\left|L_{\{2,3\}}\right|$ is even, and thus there exists a 2-element $g \in \mathbf{N}_{T}\left(H_{13}\right) \backslash L$ such that $g$ normalizes $L$ and $g^{2} \in L<H$. Now $|H: L|=14$, and as $H$ is a maximal subgroup of $T,\langle H, g\rangle=T$. Therefore, by Lemma 3.1 and Theorem 2.2 the coset graph $\Gamma=\Gamma(T, H, H g H)$ is a connected 4-transitive graph of valency 14 . Note that $H$ has a normal subgroup $N$ such that $13_{+}^{1+2} \cdot \mathbb{Z}_{12} \cong N \triangleleft L \triangleleft \mathbf{N}_{T}(L) \leq \mathbf{N}_{T}\left(H_{13}\right)=$ $N$. $\mathrm{S}_{4}$. Hence $\mathbb{Z}_{4} \cong L / N \triangleleft \mathbf{N}_{T}(L) / N \leq \mathrm{S}_{4}$, and so $\mathbf{N}_{T}(L) / N \cong \mathrm{D}_{8}$. In particular, $\mathbf{N}_{T}(L) / L \cong \mathbb{Z}_{2}$, and so by Lemma 2.3 is a unique $(T, 4)$-transitive graph of valency 14 .

To complete the proof of this lemma, we only need to prove that Aut $\Gamma=T$. Suppose that Aut $\Gamma \neq T$. Since $H$ is a maximal subgroup of $T, T$ is primitive on $V \Gamma$. Thus any group $X$ with $T<X \leq$ Aut $\Gamma$ is primitive on $V \Gamma$. Choose $X$ such that $T$ is maximal in $X$. Since $\operatorname{Aut}(T)=T$ and $T$ is primitive on $V \Gamma$, it follows that $\mathbf{N}_{\text {Aut } \Gamma}(T)=T$. Since $\Gamma$ is of valency 14 , all prime divisors of $\left|X_{\alpha}\right|$ are less than 14. As 17 exactly divides $|T|$, we have that 17 exactly divides $|X|$ and note that 17 divides $|V \Gamma|$. It then follows from the O'Nan-Scott theorem (see [24]) that $X$ is an almost simple group. Now both $T$ and $X_{\alpha}$ are maximal subgroups of $X$, and $X=T X_{\alpha}$, which is a maximal factorization of $X$. By Corollary 4 and Theorems B and $\mathrm{C}$ of [21], there exists no such triple $\left(T, X_{\alpha}, X\right)$, which is a contradiction. Therefore, Aut $\Gamma=T$, and Lemma 3.10 is proved.

\section{Proofs of the ThEOREMS}

This section is devoted to proving Theorems 1.11.4. Let $\Gamma$ be a $(G, s)$-transitive graph, where $G \leq$ Aut $\Gamma$ and $s \in\{4,5,7\}$, such that $G$ is primitive or biprimitive on $V \Gamma$. If $\Gamma$ is bipartite with parts $\Delta_{1}$ and $\Delta_{2}$, then $G_{\Delta_{1}}=G_{\Delta_{2}}$ is primitive on $\Delta_{1}$ and $\Delta_{2}$. Let

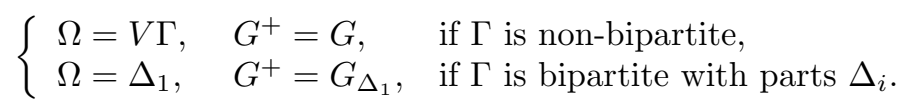

Then $G^{+}$is a primitive permutation group on $\Omega$ (see [25, Theorem 2.1]). Thus for $\alpha \in \Omega, G_{\alpha}=G_{\alpha}^{+}$is a maximal subgroup of $G^{+}$. The first lemma gives a reduction to almost simple groups. 
Lemma 4.1. Let $\Gamma$ be a graph such that $G \leq$ Aut $\Gamma$ is primitive or biprimitive on $V \Gamma$ and $\Gamma$ is $(G, s)$-transitive for some $s \in\{4,5,7\}$. Then $G^{+}$is an almost simple group, and further, either

(i) $G$ is an almost simple group, or

(ii) $\Gamma$ is the double cover of a vertex-primitive $\left(G^{+}, s\right)$-transitive graph.

Proof. By Theorem 2.2 for $\alpha \in \Omega, G_{\alpha}^{\Gamma(\alpha)}$ is a 2-transitive permutation group on $\Gamma(\alpha)$ such that $\operatorname{soc}\left(G_{\alpha}^{\Gamma(\alpha)}\right) \cong \operatorname{PSL}(2, q)$, where $q$ is a power of a prime $p$ with $|\Gamma(\alpha)|=q+1$. Since $G^{+}$is primitive, by Praeger [24, 25] and in terms of [26], $G^{+}$ is of type HA, AS, PA or TW.

Suppose first that $G^{+}$is of type HA or TW. Then $\operatorname{soc}\left(G^{+}\right)$is regular on $\Omega$. Let $P$ be the normal subgroup of $G_{\alpha}$ of order $q^{s-2}$, and let $X=\operatorname{soc}\left(G^{+}\right) \rtimes P$. Then $X \triangleleft G^{+}$, and so $1 \neq X_{\alpha}^{\Gamma(\alpha)} \triangleleft G_{\alpha}^{\Gamma(\alpha)}$. As $G_{\alpha}^{\Gamma(\alpha)}$ is 2-transitive, $X_{\alpha}^{\Gamma(\alpha)}$ is transitive on $\Gamma(\alpha)$, which is a contradiction since $|\Gamma(\alpha)|=q+1$ and $X_{\alpha}^{\Gamma(\alpha)}$ is a $p$-group.

Suppose now that $G^{+}$is of type PA, and let $N=\operatorname{soc}\left(G^{+}\right)$. Then $1 \neq N_{\alpha}^{\Gamma(\alpha)} \triangleleft$ $G_{\alpha}^{\Gamma(\alpha)}$. As $G_{\alpha}^{\Gamma(\alpha)}$ is 2-transitive, $N_{\alpha}^{\Gamma(\alpha)}$ is transitive on $\Gamma(\alpha)$, and hence $N_{\alpha}^{\Gamma(\alpha)} \geq$ $\operatorname{PSL}(2, q)$. It follows that the unique insoluble composition factor of $G_{\alpha}$ (isomorphic to $\operatorname{PSL}(2, q))$ is a composition factor of $N_{\alpha}$. However, as $G^{+}$is of type PA, $N_{\alpha}=$ $R_{1} \times \cdots \times R_{k}$, where $k \geq 2$ and $R_{1} \cong \cdots \cong R_{k}$. This is not possible. Therefore, $G^{+}$is almost simple, that is, $\operatorname{soc}\left(G^{+}\right)=T$ is a nonabelian simple group.

Assume that $G$ is not an almost simple group. It follows that $\mathbf{C}_{G}(T) \neq 1$. Since $|G| /\left|G^{+}\right|=2$ and $\mathbf{C}_{G^{+}}(T)=1$, we have that $C:=\mathbf{C}_{G}(T) \cong \mathbb{Z}_{2}$. Now $C \triangleleft G$, and it then follows that $G=G^{+} \times C$. Let $\Sigma=\Gamma_{C}$, the quotient of $\Gamma$ induced by $C$. Then $\Gamma$ is the standard double cover of $\Sigma$. Further, $G^{+} \cong G / C \leq$ Aut $\Sigma, G / C$ is primitive on $V \Sigma$, and by [24, Theorem 4.1], $\Sigma$ is $(G / C, s)$-transitive.

Thus, to complete the proofs of Theorems 1.3 and 1.4, we only need to consider almost simple groups. So we assume that $G$ is an almost simple group with socle $T=\operatorname{soc}(G)=\operatorname{soc}\left(G^{+}\right)$, and that $\Gamma$ is a $(G, s)$-transitive graph with $s \in\{4,5,7\}$. We analyse the finite nonabelian simple groups class by class.

Lemma 4.2. Assume that $T=\operatorname{soc}(G) \cong \mathrm{A}_{n}$ for some $n \geq 5$. Then $n=6$, and $\Gamma$ is a bipartite 5-transitive cubic graph, which is isomorphic to Tutte's 8-Cage (see Example 3.6).

Proof. Assume that $n=6$. Then $T \cong \operatorname{PSL}(2,9)$, and $\operatorname{PSL}(2,9) \leq G \leq \operatorname{P\Gamma L}(2,9)$. By information given in the Atlas [8, $\operatorname{P\Gamma L}(2,9)$ has a maximal subgroup $\mathrm{S}_{4} \times \mathbb{Z}_{2}$, and further we may conclude that the pair $\left(\mathrm{P} \Gamma \mathrm{L}(2,9), \mathrm{S}_{4} \times \mathbb{Z}_{2}\right)$ gives rise to the smallest graph $\Gamma$ constructed in Example 3.6, which is bipartite, cubic and 5transitive.

Suppose that $n \neq 6$. Then either $G=G^{+}$, or $G^{+}=T$ and $G \cong \mathrm{S}_{n}$. In particular, $G^{+} \cong \mathrm{A}_{n}$ or $\mathrm{S}_{n}$. Now $G^{+}$naturally acts on $\Omega=\{1,2, \ldots, n\}$, and $G_{\alpha}^{+}$is a maximal subgroup of $G^{+}$. Consider the $G_{\alpha}^{+}$-action on $\Omega$. Note that $G_{\alpha}^{+}$has the form of $H$ given in Table 3 of Theorem 2.2, and in particular, the unique insoluble composition factor of $G_{\alpha}^{+}$is PSL $(2, q)$. By the O'Nan-Scott theorem (see [21, p. 130]), we may conclude that $n=p^{2}$ and $G_{\alpha}^{+}=\operatorname{AGL}(2, p) \cap G^{+}=\left(\mathbb{Z}_{p}^{2} \rtimes\left(\mathbb{Z}_{p-1}\right.\right.$. PSL $\left.\left.(2, p) \cdot \mathbb{Z}_{2}\right)\right) \cap G^{+}$. Suppose that $T_{\alpha}=\mathbb{Z}_{p}^{2} \rtimes\left(\mathbb{Z}_{p-1} \cdot \operatorname{PSL}(2, p) \cdot \mathbb{Z}_{2}\right)=\operatorname{AGL}(2, p)$. Then there exists an involution $\sigma$ of $\mathrm{S}_{n} \backslash \mathrm{A}_{n}$ which normalizes $T_{\alpha}$. However, by the O'Nan-Scott theorem, $\left\langle T_{\alpha}, \sigma\right\rangle$ is not a subgroup of $\mathrm{S}_{n}$, which is a contradiction. Thus $T_{\alpha}=$ $\mathbb{Z}_{p}^{2} \rtimes\left(\mathbb{Z}_{p-1}\right.$. PSL $\left.(2, p)\right)$. If $\Gamma$ is non-bipartite, then $\Gamma$ is $(T, s)$-transitive, which is a 
contradiction since $T_{\alpha}$ does not satisfy Theorem 2.2 If $\Gamma$ is bipartite, then $G^{+}=T$ and so $G_{\alpha}^{+}=T_{\alpha}=\mathbb{Z}_{p}^{2} \rtimes\left(\mathbb{Z}_{p-1}\right.$. $\left.\operatorname{PSL}(2, p)\right)$, which is again a contradiction since $G^{+}$ does not satisfy Theorem 2.2

Lemma 4.3. Assume that $T=\operatorname{soc}(G)$ is a sporadic simple group. Then $T=\mathrm{M}_{12}$, $\mathrm{J}_{3}, \mathrm{Ru}$, Th or $\mathrm{M}$, and Theorems 1.3 and 1.4 hold.

Proof. For a vertex $\alpha$ of $\Gamma, T_{\alpha}$ satisfies Theorem 2.2. Thus, by information given in the Atlas 8] and the recent result in [23, 38, the pair $\left(T, T_{\alpha}\right)$ is one of the following:

$\left(\mathrm{M}_{12}, \mathbb{Z}_{3}^{2} \rtimes \mathrm{GL}(2,3)\right) ;\left(\mathrm{M}_{22}, \mathbb{Z}_{2}^{4} \rtimes \mathrm{PSL}(2,4) . \mathbb{Z}_{2}\right) ;\left(\mathrm{M}_{23}, \mathbb{Z}_{2}^{4} \rtimes \mathrm{GL}(2,4) . \mathbb{Z}_{2}\right) ;$

$\left(\mathrm{J}_{3}, \mathbb{Z}_{2}^{4} \rtimes \mathrm{GL}(2,4)\right) ;\left(\mathrm{McL}, \mathbb{Z}_{3}^{4} \rtimes \mathrm{M}_{10}\right) ;\left(\mathrm{He}, \mathbb{Z}_{7}^{2} \rtimes \mathrm{SL}(2,7)\right) ;$

$\left(\mathrm{Ru}, \mathbb{Z}_{5}^{2} \rtimes \mathrm{GL}(2,5)\right) ;\left(\mathrm{Th}, \mathbb{Z}_{5}^{2} \rtimes \mathrm{GL}(2,5)\right) ;\left(\mathrm{M}, \mathbb{Z}_{13}^{2} \rtimes\left(\mathbb{Z}_{4}\right.\right.$. $\left.\left.\mathrm{PSL}(2,13) . \mathbb{Z}_{2}\right)\right)$.

Suppose that $T=\mathrm{M}_{22}$ or $\mathrm{M}_{23}$. Then the graph $\Gamma$ has valency 5 , and $\left|T: T_{\alpha}\right|$ is odd, which is a contradiction. Next suppose $\left(T, T_{\alpha}\right)=\left(\mathrm{McL}, \mathbb{Z}_{3}^{4} \rtimes \mathrm{M}_{10}\right)$. If there exists such a graph $\Gamma$, then $T_{\alpha} \cap T_{\alpha}^{g}=T_{3} \rtimes \mathrm{Q}_{8}$ where $T_{3} \cong \mathbb{Z}_{3}^{4}$ is a Sylow 3 -subgroup of $T$ and $g$ normalizes $T_{3} \rtimes \mathrm{Q}_{8}$. Thus we have $\mathbf{N}_{T}\left(T_{3}\right)=T_{3} \rtimes[16]$. However, by the Atlas [8, this is not possible. An argument for $T=$ He using Sylow 7-subgroups, which is analogous to the argument for McL using Sylow 3-subgroups, proves that $T \neq$ He.

The pair $\left(T, T_{\alpha}\right)=\left(\mathrm{M}_{12}, \mathbb{Z}_{3}^{2} \rtimes \mathrm{GL}(2,3)\right)$ gives rise to the bipartite graph $\Gamma$ described in Lemma 3.7] such that Aut $\Gamma=\operatorname{Aut}\left(\mathrm{M}_{12}\right)=\mathrm{M}_{12} \cdot \mathbb{Z}_{2}$. The pair $\left(T, T_{\alpha}\right)=$ $\left(\mathrm{J}_{3}, \mathbb{Z}_{2}^{4} \rtimes \mathrm{GL}(2,4)\right)$ gives rise to the Weiss graph (see Lemma 3.8). The pair $\left(\mathrm{Ru}, \mathbb{Z}_{5}^{2} \rtimes\right.$ GL $(2,5)$ ) gives rise to the Stroth-Weiss graph, one of the graphs constructed in Lemma 3.10 while the pairs (Th, $\left.\mathbb{Z}_{5}^{2} \rtimes \mathrm{GL}(2,5)\right)$ and $\left(\mathrm{M}, \mathbb{Z}_{13}^{2} \rtimes\left(\mathbb{Z}_{4}\right.\right.$. PSL $\left.\left.(2,13) . \mathbb{Z}_{2}\right)\right)$ give rise to the other two graphs constructed in Lemma 3.10

We note that, by Lemma 3.10 and Lemma 4.3, we have completed the proof of Theorem 1.1.

Assume next that $\operatorname{soc}(G)$ is a classical simple group of Lie type. Note that $H$ is a maximal local subgroup of $G^{+}$. All possibilities for $H$ are described in [16], and we will analyse the classification in [16] to complete this case. For convenience, we first deal with some small groups, using information given in the Atlas [8].

Lemma 4.4. The socle $T=\operatorname{soc}(G)$ of $G$ is not one of the following groups:

(1) $\operatorname{PSL}(3,2), \operatorname{PSL}(4,2), \operatorname{PSL}(5,2), \operatorname{PSL}(4,3)$;

(2) $\operatorname{PSU}(4,2), \operatorname{PSU}(5,2), \operatorname{PSU}(6,2), \operatorname{PSU}(3,3), \operatorname{PSU}(4,3)$;

(3) $\operatorname{PSp}(6,2), \operatorname{PSp}(8,2), \operatorname{PSp}(4,3)(\cong \operatorname{PSL}(4,2)), \operatorname{PSp}(6,3)$;

(4) $\mathrm{O}_{8}^{+}(2), \mathrm{O}_{8}^{-}(2), \mathrm{O}_{10}^{+}(2), \mathrm{O}_{10}^{-}(2), \mathrm{O}_{7}(3), \mathrm{O}_{8}^{+}(3), \mathrm{O}_{8}^{-}(3)$.

Proof. For the groups listed in (1), from information given in the Atlas [8, if $T=\operatorname{PSL}(3,2)$, then $H=\mathrm{S}_{4}$, but $T$ is 2-transitive on $[T: H]$, a contradiction; while if $T$ is one of the other groups in (1), there is no a subgroup $H$ of $T$ satisfying Theorem 2.2.

Now consider the groups listed in (2). By the Atlas 8], only $\operatorname{PSU}(4,2)$ and $\operatorname{PSU}(4,3)$ have subgroups satisfying Theorem 2.2 If $T=\operatorname{PSU}(4,2)$, then $H$ is isomorphic to $\left[2^{4}\right] \rtimes \mathrm{A}_{5}$ or $\left[3^{3}\right] \rtimes 2 \mathrm{~A}_{4}$. Thus the valency $k=5$ or 4 , and $K \cong\left(\left[2^{6}\right] .3\right) .2$ or $\left[3^{4}\right]$.[4], but $\operatorname{PSU}(4,2)$ has no such subgroups. If $T=\operatorname{PSU}(4,3)$, then $H$ is isomorphic to $3^{4} \rtimes \mathrm{A}_{6}$ or $\left[3^{5}\right] .2 \mathrm{~S}_{4}$. Thus $K$ should be isomorphic to [3 $\left.{ }^{6}\right]$.[8], but $\operatorname{PSU}(4,3)$ has no such subgroups.

Similarly, from the information given in the Atlas [8], we may easily conclude that $T$ is not one of the groups in (3) or (4). 
Next we check subgroups in $\mathcal{C}_{i}$ for $1 \leq i \leq 8$ described in [16]. Observing the form of the point-stabilizer in $G$, by Sections $4.2,4.4,4.5,4.7$ and 4.8 and by Lemma 4.4 we may conclude that $H$ is not in $\mathcal{C}_{2} \cup \mathcal{C}_{4} \cup \mathcal{C}_{5} \cup \mathcal{C}_{7} \cup \mathcal{C}_{8}$. Thus we only need to consider the remaining three cases.

Lemma 4.5. Assume that $T=\operatorname{soc}(G)$ is a classical simple group of Lie type. Then $T=\mathrm{A}_{6}, \operatorname{PSL}(3,3), \operatorname{PSL}(3, q), \operatorname{PSp}\left(4,2^{m}\right), \operatorname{PSL}(2, p)$, or $\operatorname{PSp}(4, p)$ with $p \equiv \pm 1$ $(\bmod 8)$, and $\Gamma$ is one of the graphs given in Tables 1 and 2.

Proof. First assume that $H$ is a subgroup of $G$ in $\mathcal{C}_{1}$, namely a reducible subgroup. Checking Section 4.1 of [16] and using Lemma 4.4 we find that one of the following holds:

$T=\operatorname{PSL}(3, q)$ and $T_{\alpha}=\left[q^{2}\right] \cdot \mathbb{Z}_{\frac{q-1}{(q-1,3)}} \operatorname{PGL}(2, q)$ (see [16, Proposition 4.1.17]);

$T=\operatorname{PSp}(4, q)$ and $T_{\alpha}=\left[q^{3}\right]$. GL $(2, q)$ where $q$ is a 2-power (see [16, Proposition 4.1.19]).

We note that although for $T=\operatorname{PSU}(4, q)$ where $q$ is a 3 -power, $T$ has a subgroup $M$ which has a normal subgroup $N$ of order $q^{5}$ and has a unique insoluble composition factor isomorphic to $\operatorname{PSL}(2, q), M / N \neq \mathrm{GL}(2, q)$. A similar situation happens for the group $\operatorname{PSL}(4, q)$. Therefore, $T$ is isomorphic to $\operatorname{PSL}(3, q)$ or $\operatorname{PSp}(4, q)$. The former gives rise to the generalized 3-gon graphs, and the latter gives rise to the generalized 4-gon graphs described in Section 2.

Now assume that $H$ is a subgroup of type $\mathcal{C}_{3}$, that is a field extension subgroup. Then by Section 4.3 of [16], we may conclude that $T=\operatorname{PSL}\left(2,3^{2}\right) \cong \mathrm{A}_{6}$, and $H \cap T \cong \mathrm{PGL}(2,3) \cong \mathrm{S}_{4}$. This case gives rise to one of the graphs $\Gamma$ constructed in Example 3.6 such that Aut $\Gamma=\operatorname{P\Gamma L}\left(2,3^{2}\right)$.

Finally, we assume that $H$ is a subgroup of $G$ in $\mathcal{C}_{6}$, namely a symplectic-type normalizer. Checking Section 4.6 of [16] and by Lemma 4.4, we may conclude that one of the following cases holds:

(1) $T=\operatorname{PSL}(3,3)$, and $H \cap T \cong \operatorname{PSL}(2,3) . \mathbb{Z}_{2} \cong \mathrm{S}_{4}$ (see [16] Proposition 4.6.5]);

(2) $T=\operatorname{PSL}(2, p)$ with $p \equiv \pm 1(\bmod 8)$ and $H \cong \mathrm{S}_{4}$ (see [16, Proposition 4.6.7]);

(3) $T=\operatorname{PSp}(4, p)$ with $p \equiv \pm 1(\bmod 8)$, and $H \cong \mathbb{Z}_{2}^{4} \cdot\left(\Omega_{4}^{-}(2) \cdot \mathbb{Z}_{2}\right) \cong \mathbb{Z}_{2}^{4} \cdot \operatorname{GL}(2,4)$ (see [16, Proposition 4.6.9]).

Case (1) gives rise to the Wong-graph described in Example 3.3. Case (2) gives rise to the Biggs-Hoare-graphs $\Gamma$ described in Example 3.5 such that Aut $\Gamma=$ $\operatorname{PGL}(2, p)$; if further $p \equiv \pm 1(\bmod 16)$, then Case $(2)$ gives rise to the Wonggraphs described in Example 3.4 Case (3) gives rise to the graphs constructed in Lemma 3.9. This completes the proof of the lemma.

We remark that, by Lemma 3.9 and Lemma 4.5 we have completed the proof of Theorem 1.2.

Finally, we deal with exceptional simple groups of Lie type.

Lemma 4.6. If $T$ is an exceptional simple group of Lie type, then $T=\mathrm{G}_{2}(q)$ with $q=3^{m}$ for some $m \geq 1$, and $T_{\alpha} \cong\left[q^{5}\right] \rtimes \mathrm{GL}(2, q)$, as in Theorem 1.4.

Proof. Let $T=\operatorname{soc}(G)$ be an exceptional simple group of Lie type over $G F\left(t^{f}\right)$ where $t$ is a prime. Let $H$ be a maximal subgroup of $T$ which is isomorphic to $T_{\alpha}$.

Assume first that $T=\mathrm{F}_{4}(q), \mathrm{E}_{i}(q)$ for $i \in\{6,7,8\}$, or ${ }^{2} \mathrm{E}_{6}(q)$.

Let $E$ be a minimal normal subgroup of $H$, and let $\operatorname{InnDiag}(T)$ be the group generated by all inner and diagonal automorphisms of $T$. Then $E$ is elementary abelian and $H=\mathbf{N}_{T}(E)$. By [5], there are four cases to be considered: 
(i) $E<\operatorname{InnDiag}(T)$ and $H$ is of maximal rank;

(ii) $E<\operatorname{InnDiag}(T)$ and $H$ is not of maximal rank;

(iii) $E \nless \operatorname{InnDiag}(T)$;

(iv) $t|| E \mid$, so that $H$ is a maximal parabolic subgroup of $G$.

Suppose that case (i) holds. Then all possibilities for $H$ are listed in [5, Tables 5.1 and 5.2]. We may easily conclude that no subgroups $H$ satisfy Theorem 2.2, which is a contradiction.

Suppose that case (ii) holds. Then all possibilities are listed in [5 Table 1]. Checking the list, we find that $H$ has a nonabelian simple composition factor not isomorphic to $\operatorname{PSL}(2, q)$, which is a contradiction.

Suppose that case (iii) holds. Then $E \cap \operatorname{InnDiag}(T)=1$. It follows from 12 . Sections 7 and 9] that $H \cap T=\mathbf{C}_{T}(\alpha) \neq 1$ for some outer automorphism $\alpha$ of $T$ of prime order. Therefore, by [2, Section 19] and [5, Proposition 2.7], either $\mathbf{C}_{T}(\alpha)$ is a group of Lie type over a maximal subfield of $G F\left(t^{f}\right)$, or $\mathbf{C}_{T}(\alpha)$ is ${ }^{2} \mathrm{~F}_{4}(q),{ }^{2} \mathrm{E}_{6}\left(q^{1 / 2}\right)$, $\mathrm{C}_{4}(q)$ with $q$ odd, or $\mathrm{F}_{4}(q)$. Each of them contains an insoluble composition factor not isomorphic to $\operatorname{PSL}(2, q)$, which is a contradiction.

Suppose that case (iv) holds. Then $H$ is an extension of a $t$-group by the Chevalley group determined by a maximal subdigram of the Dynkin diagram of $T$. It follows that either $H$ contains a nonabelian composition factor not isomorphic to $\operatorname{PSL}(2, q)$, or $T=\mathrm{F}_{4}(2)$. The former is not the case. By the Atlas $[8], \mathrm{F}_{4}(2)$ has no maximal subgroup satisfying Theorem 2.2 which is a contradiction.

Now consider the remaining exceptional simple groups $T$ of Lie type. All maximal subgroups of such groups $T$ are completely known: 29] for $T=\mathrm{Sz}(q)$, 22] for $T={ }^{2} \mathrm{~F}_{4}\left(q^{2}\right)$, 15] for $T={ }^{2} \mathrm{G}_{2}(q)$, and [14 for $T={ }^{3} \mathrm{D}_{4}(q)$. Checking these lists of maximal subgroups of $T$, we may conclude that $T=\mathrm{G}_{2}(q)$ with $q=3^{m}$ and $T_{\alpha}=\left[q^{5}\right] \rtimes \mathrm{GL}(2, q)$. This gives rise to the generalized 6 -gon graphs described in Section 3. The proof of the lemma is completed.

Combining Lemmas 4.14.6 we have completed the proofs of Theorem 1.3 and Theorem 1.4.

\section{REFERENCES}

[1] M. Aschbacher, Overgroups of Sylow Subgroups in Sporadic Groups, Memoirs Amer. Math. Soc., 343 (1986). MR 87e:20037

[2] M. Aschbacher and G. M. Seitz, Involutions in Chevalley groups over fields of even order, Nagoya Math. J. 63 (1976), 1-91; Correction, Nagoya Math. J. 72 (1978), 135-136. MR 54:10391 MR 80b:20058

[3] N. Biggs, Algebraic Graph Theory, Cambridge Univ. Press, New York (2th Ed. 1993). MR 95h:05105

[4] N. Biggs and M. Hoare, The sextet construction for cubic graphs, Combinatorica 3 (1983), 153-165. MR 85a:05038

[5] A. M. Cohen, M. W. Liebeck, J. Saxl and G. M. Seitz, The local maximal subgroups of the exceptional groups of Lie type, Proc. London Math. Soc. (3), 64 (1992), 21-48. MR 92m:20012

[6] M. Conder, An infinite family of 5-arc-transitive cubic graphs, Ars Combin. 25(A) (1988), 95-108. MR 89f:05092

[7] M. Conder and C. Walker, The infinitude of 7-arc transitive graphs, J. Algebra 208 (1998), 619-629. MR 99j:05089

[8] J. H. Conway, R. T. Curtis, S. P. Norton, R. A. Parker and R. A. Wilson, Atlas of Finite Groups, Clarendon Press, Oxford, 1985. MR 88g:20025

[9] A. Gardiner, Arc transitivity in graphs, Quart. J. Math. Oxford (2) 24 (1973), 399-407. MR 48:1973 
[10] A. Gardiner, Doubly primitive vertex stabilizers in graphs, Math. Z. 135 (1974), 257-266. MR 54:143

[11] D. Goldschmidt, Automorphisms of trivalent graphs, Ann. Math. 111 (1980), 377-406. MR 82a:05052

[12] D. Gorenstein and R. Lyons, The local structure of finite groups of characteristic 2 type, Memoirs of Amer. Math. Soc., 42 (1983), No. 276. MR 84g:20025

[13] A. A. Ivanov and S. V. Shpectorov, Applications of group amalgams to algebraic graph theory, in Investigations in Algebraic Theory of Combinatorial Objects, Ed. by I. Faradzev, 417-441, (Kluwer Academic Publ. 1994). MR 95m:05122

[14] P. Kleidman, The maximal subgroups of the Steinberg triviality groups ${ }^{3} \mathrm{D}_{4}(q)$ and their automorphism groups, J. Algebra 115 (1988), 182-199. MR 89f:20024

[15] P. Kleidman, The maximal subgroups of the Chevalley groups $\mathrm{G}_{2}(q)$ with $q$ odd, the Ree groups ${ }^{2} \mathrm{G}_{2}(q)$ and their automorphism groups, J. Algebra 117 (1988), 30-71. MR 89j:20055

[16] P. Kleidman and M. Liebeck, The Subgroup Structure of the Finite Classical Groups, London Math. Soc. Lecture Notes, Series 129, Cambridge University Press, Cambridge (1990). MR 91g:20001

[17] C. H. Li, A family of quasiprimitive 2-arc-transitive graphs which have non-quasiprimitive full automorphism groups, Europ. J. Combin. 19 (1998), 499-502. MR 99e:05066

[18] C. H. Li, Finite s-arc transitive graphs of prime-power order, Bull. London Math. Soc. (to appear).

[19] C. H. Li, On finite s-transitive graphs of odd order, J. Combin. Theory Ser. B, 81 (2001), 307-317.

[20] C. H. Li, C. E. Praeger, A. Venkatech and S. Zhou, Finite locally quasiprimitive graphs, Discrete Math. (to appear).

[21] M. Liebeck, C. E. Praeger and J. Saxl, The Maximal Factorizations of the Finite Simple Groups and Their Automorphism Groups, Memoirs of Amer. Math. Soc., 86 (1990). MR 90k:20048

[22] G. Malle, The maximal subgroups of ${ }^{2} \mathrm{~F}_{4}\left(q^{2}\right)$, J. Algebra 139 (1991), 52-69. MR 92d:20068

[23] U. Meierfrankenfeld and S. V. Shpektorov, The maximal 2-local subgroups of the Monster and Baby Monster, in preparation.

[24] C. E. Praeger, An O'Nan-Scott theorem for finite quasiprimitive permutation groups and an application to 2-arc transitive graphs, J. London. Math. Soc. 47 (1992), 227-239. MR 94f:05068

[25] C. E. Praeger, On a reduction theorem for finite bipartite 2-arc transitive graphs, Australs. J. Combin. 7 (1993), 21-36. MR 93m:05091

[26] C. E. Praeger, Finite transitive permutation groups and finite vertex-transitive graphs, Graph Symmetry: Algebraic Methods and Applications, NATO Ser. C, 497 (1997), pp. 277-318. MR 98h:05093

[27] G. O. Sabidussi, Vertex-transitive graphs, Monatsh Math. 68 (1964), 426-438. MR 31:91

[28] G. Stroth and R. Weiss, A new construction of the group Ru, Quart. J. Math. Oxford Ser. (2) 41 (1990), 237-243. MR 91m:20025

[29] M. Suzuki, On a class of doubly transitive groups, Ann. Math. 75 (1962), 104-145. MR 25:112

[30] M. Suzuki, Group Theory I, Springer-Verlag, New York, 1982. MR 82k:20001c

[31] J. Tits, Sur la trialité at certains groupes qui s'en déduisent, Publ. Math. IHES 2 (1959), $14-60$.

[32] W. T. Tutte, A family of cubical graphs, Proc. Cambridge Phil. Soc. 43 (1947), 459-474. MR 9:97g

[33] R. Weiss, Groups with a $(B, N)$-pair and locally transitive graphs, Nagoya Math. J. 74 (1979), 1-21. MR 81j:05067

[34] R. Weiss, s-transitive graphs, In Algebraic Methods in Graph Theory, Vol. 2 (1981), 827-847. MR 83b:05071

[35] R. Weiss, The nonexistence of 8-transitive graphs, Combinatorica 1 (1981), 309-311. MR 84f:05050

[36] R. Weiss, A characterization of the group $\hat{M}_{12}$, Algebras Groups Geom. 2 (1985), 555-563. MR 87k:20010

[37] R. Weiss, A characterization and another construction of Janko's group $J_{3}$, Trans. Amer. Math. Soc. 298 (1986), 621-633. MR 88g:20028 
[38] R. A. Wilson, The maximal subgroups of the Baby Monster, I, J. Algebra 211 (1999), 1-14. MR 2000b:20016

[39] W. J. Wong, Determination of a class of primitive permutation groups, Math. Z. 99 (1967), 235-246. MR 35:5502

Department of Mathematics and Statistics, The University of Western Australia, Nedlands, WA 6907, Australia

E-mail address: li@maths.uwa.edu.au 